\title{
A weak fault diagnosis method for rotating machinery based on compressed sensing and stochastic resonance
}

\author{
Peiming Shi ${ }^{1}$, Xiaojie $\mathrm{Ma}^{2}$, Dongying $\mathrm{Han}^{3}$ \\ ${ }^{1,2}$ School of Electrical Engineering, Yanshan University, Qinhuangdao, 066004, China \\ ${ }^{3}$ School of Vehicles and Energy, Yanshan University, Qinhuangdao, 066004, China \\ ${ }^{1}$ Corresponding author \\ E-mail: ${ }^{1}$ spmysu@qq.com, ${ }^{2} 286123264 @ q q . c o m,{ }^{3}$ dongying.han@163.com
}

Received 14 August 2018; received in revised form 28 November 2018; accepted 25 December 2018 DOI https://doi.org/10.21595/jve.2018.20140

Check for updates

Copyright $(C) 2019$ Peiming Shi, et al. This is an open access article distributed under the Creative Commons Attribution License, which permits unrestricted use, distribution, and reproduction in any medium, provided the original work is properly cited.

\begin{abstract}
Vibration signals used for rotating machinery fault diagnosis often constitute large amount of data. It is a big challenge to extract faults feature information from these data. Recently, a new sampling framework called compressed sensing has been proposed, which enables the recovery from a small set of measured data if the signals are sparse or compressible. In reality, the sparseness of the signals is not very well due to noise, so it is difficult and unavailing to recover the whole signal. Thus, a new mechanical fault diagnosis method is proposed in this paper. First, the machine fault vibration signals are pretreated by stochastic resonance. By this way, the fault signal drowned by noise is amplified and the sparseness of the signals is enhanced, which make it possible to apply compressed sensing. Second, fault features are extracted directly from the compressed data without recovering completely, which reduces the dimensionality of the measurement data and the complexity of algorithm. Finally, the effectiveness of the proposed method is proved by the experiments.
\end{abstract}

Keywords: rotating machinery, weak fault diagnosis, feature extraction, compressed sensing, stochastic resonance.

\section{Introduction}

Rotating machinery is one of the most important equipment in industrial fields. Condition monitoring and fault diagnosis is important to ensure safe and stable operation for rotating machinery equipment. Vibration based method is the most effective to monitor the faults of rotating machinery $[1,3]$. The acquisition of vibration signal is usually based on the Shannon sampling theory which requires the least twice sampling rate of the signal. With the rapid growing demand of information for complex mechanical systems, the large amount of data has been produced. For example, a wind turbine condition monitoring system, which has been installed in an operational Vestas V47 wind turbine, captures 2-TB data/month [4]. The huge data makes a heavy burden on the transmission, storage and processing of signals. Recently, a new sampling theory compressed sensing (CS) is proposed, which pointed out that the signal can be recovery from far few measurements than the Shannon sampling theory if the signal is sparse or compressible [5-7].

The CS has been applied in many areas. Many scholars have begun to attempt applying the CS technology to mechanical vibration signal. For example, Wang et al. developed a compressed detection strategy of roller bearing fault based on multiple down-sampling method [8]; Du et al. presented a new mechanical feature identification method, which extracted fault features directly from compressed domain [9]; Tang et al. proposed a compressed sensing frame of characteristics harmonics to detect roller bearing faults [10]; Hang et al. proposed a bearing fault diagnosis method under fluctuate conditions based on compressed sensing theory [11]; Tang developed a sparse classification method for rotating machinery faults based on compressed sensing strategy [12]. These studies have proved the effectiveness of CS in mechanical fault detection. However, weak fault feature identification of vibration signals mingled with heavy noise is still a challenging issue in rotating machinery fault diagnosis. In order to solve this problem, a new mechanical 
feature identification method is proposed in this paper based on the CS theory and compressed feature detection techniques. In the method, we use stochastic resonance (SR) to pre-processing the vibration signal.

$\mathrm{SR}$ is a nonlinear phenomenon which means that a signal can be improved when the noise level is increased or when specific noise is added to the system. Since the SR concept was proposed by Benzi et al. in 1981 [13], it has been widely applied in signal processing, physics, biology, large mechanical fault diagnosis, and other fields [14-16]. In recent years, the application of SR in mechanical fault diagnosis has been widely used. For example, Han et al. designed a SR model with parameter compensation and wavelet transform to detect the signal characteristics of a multi-frequency weak signal [17]; Li et al. present a multi-stable SR method to enhance the processing capability of SR for weak [18]; Qin et al. built an adaptive SR method based on dyadic wavelet transform and least squares, which increased the calculation speed and improve the weak feature detection performance [19]; Zhang et al. proposed a bearing fault enhancement detection method based on signal cepstrum pre-whitening and stochastic resonance [20]. In contrast to traditional de-noising methods, SR utilizes noise instead of eliminating it, to improve the signal-to noise ratio and allow for detection of weak signals. In our method, the sparsity and the failure feature are enhanced after treated by SR and it becomes suitable for the CS. Then, the CS theory is used to compressed sample. At last, faults features are directly extracted from the compressed data. Its main advantage is that weak fault features mixed in noise are detected directly in the compressed measurements domain without sacrificing accuracy. The experiments are performed to prove the reliability and effectiveness of the proposed method.

This paper is organized as follows. In Section 2, we introduce the theory of the CS and stochastic resonance. Then, the algorithm framework for machine fault diagnosis is presented in detail. Section 3 made two experiments and the results are presented, which demonstrates that the method is valid to many kinds of mechanical fault detections. Finally, the conclusions are given in Section 4.

\section{The method for weak feature identification}

In this section, the basic concepts of compressed sensing and stochastic resonance are introduced, and the weak fault diagnosis method for rotating machinery is presented.

\subsection{Compressed sensing}

CS is a new sampling method, which is proposed to solve the big data problem. In CS, the signal can be recovered from far fewer samples than required by the Shannon sampling theory [5]. There are two conditions for CS Technology. First, the signal must be sparse in some domain. Second, the signal is incoherence, which is applied through the isometric property and is sufficient for sparse signals. The matrix representation of compressed sensing is shown in Fig. 1.

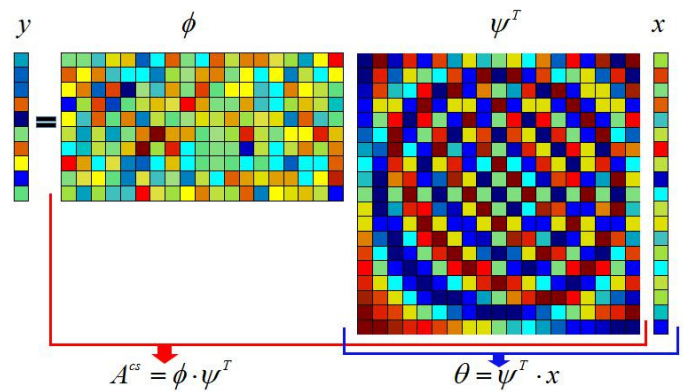

Fig. 1. The matrix representation of compressed sensing

For the original signal, the first step is to transform the original signal to a sparse signal: 
$x=\psi \cdot \theta, \quad \theta=\psi^{T} \cdot x$,

where $x$ is $N \times 1$ vector; $\psi$ is sparse matrix, which is an orthogonal matrix of $N \times N ; \theta$ is sparse vector of $N \times 1$, which can be said $K$-sparse if the vector contains only $K$ non-zero values $(K \ll N)$.

Then, it is the dimension reduction and can be represented as:

$y=\phi \cdot \theta=\phi \cdot \psi^{T} \cdot x=A^{C S} \cdot x$,

where $\phi$ is the observation matrix of $M \times N,(M \ll N)$; The compressed data $y$ is $M \times 1$ vector; $A^{C S}$ is the matrix of $M \times N\left(A^{C S}=\phi \cdot \psi^{T}\right)$.

The most important step of signal reconstruction is the recovery of $\theta$. The solution can be shown as follows:

$\min \|\theta\|_{0}$ subject to $y=\phi \cdot \theta$.

Owing to the sparsity of $\theta$ and the incoherence between $\psi$ and $\phi$, the vector $\theta$ can be reconstructed with a high probability based on a reconstruction algorithm. After that, the original signal can be obtained by Eq. (1).

The dimension of the vector $\mathrm{y}$ is determined by:

$M \geq C \mu^{2} K \log N$,

where $C$ is a positive constant; and $\mu$ is the correlation between $\psi$ and $\phi$, which can be represented as:

$\mu=\sqrt{N} \cdot \max _{i, j}\left(\left|\left\langle\phi_{i}, \psi_{j}\right\rangle\right|\right)$,

where $\phi$ is the random matrix, which is uncorrelated with any other matrix. The sparse matrices are usually the Fourier, DCT or wavelet matrices. In the following experiments, random matrices and Fourier matrices were used as observation matrices and sparse matrices, respectively.

\subsection{Stochastic resonance}

Stochastic resonance occurs in a nonlinear system with noise and periodic input signal, which can be described by the Langevin equation:

$\dot{x}=-U^{\prime}(x)+s(t)+\Gamma(t)$,

where $s(t)$ is the periodic input signal; $\Gamma(t)$ denotes a Gaussian white noise with a zero-mean, which satisfies $\langle\Gamma(t)\rangle=0$ and $\langle\Gamma(t), \Gamma(0)\rangle \geq 2 D \delta(t)$, where $D$ is the noise intensity and $\delta(t)$ is the unit impulse function; $U(x)$ is the traditional bistable potential function and is shown in Fig. 2 . The barrier parameters $a$ and $b$ in $U(x)$ are positive real parameters:

$U(x)=-\frac{a}{2} x^{2}+\frac{b}{4} x^{4}, a>0, \quad b>0$.

According to Eq. (7), the barrier height is $\Delta U=a^{2} / 4 b$, and the steady state of the system is at:

$x= \pm \sqrt{\frac{a}{b}}$

Then, Eq. (6) can be written as: 
$\dot{x}=a x-b x^{3}+s(t)+\Gamma(t)$.

The periodic signal can be presented as $s(t)=A \cos 2 \pi F t$. When the periodic signal and the noise are acting at the same time, the periodic signal will induce a periodic change to the system potential wells and synchronize the noise-induced switching.

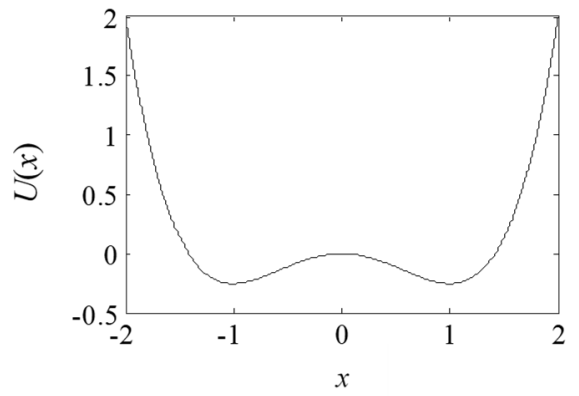

Fig. 2. The potential function $U(x)(a=b=1)$

In the case that the amplitude of $s(t)$ is smaller than the critical value of the barrier height $A_{C}=\sqrt{4 a^{3} / 27 b}$, the particles can cross from the original potential well to another potential well at Kramer's rate $r_{k}=(a / \sqrt{2} \pi) \exp (-\Delta U / D)$. What's more, the system output is switched between the two potential wells by the frequency $\omega$ of the signal; the switching speed synchronizes the output signal with the weak periodic signal. When the transition rate matches the period of the input signal $\left(2 / r_{k}=1 / F\right)$, the frequency $\omega$ is equal to $F$, the component of the frequency $F \mathrm{n}$ the output $x(t)$ is enhanced. That is why stochastic resonance can strengthen the weak signal and improve the sparsity. The transition of particles between two potential wells is shown in Fig. 3.

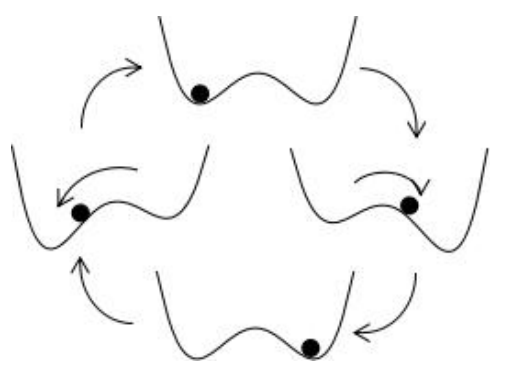

Fig. 3. The diagram of the transition between two potential wells of stochastic resonance

\subsection{The proposed method}

CS has been applied in many areas, such as nuclear magnetic resonance, image processing, etc. But CS is still little application in mechanical fault diagnosis field. The main problem is the poor sparsity of mechanical signal caused by the noise. Even worse, the useful fault feature information may be buried in noise, especially at the initial fault stages. As for the fault detection section, the traditional idea is to recover the whole signal from the compressed data, and then extract the fault characteristics from the whole signal, just like the workflow shown in Fig. 4(a). For the real vibration signals, this process is tedious and impossible because of the complex noise. So, there is a question, if it is possible to extract the faults directly from the compressed data rather than reconstruct the whole signal, just like the process shown in Fig. 4(b). The reconstruct methods of the signal are usually consisted of greedy pursuit's algorithm, convex relaxation algorithm and combinatorial algorithm [21]. In this paper, Compressed Sampling Matching Pursuit (CSMP) 
algorithm is applied to reconstruct the signal, which is one kind of greedy pursuit's algorithm. CSMP invokes the idea of iteratively to reconstruct the signal [22]. The current approximation induces a residual in each iteration. The samples are updated to reflect the current residual with the processing of algorithm. These samples construct a proxy for the residual, and it is useful to identify the large components in the residual. This step yields a tentative support for the next approximation. The samples to estimate the approximation on the support set. This process is repeated until we have found the recoverable energy in the signal. What's more, CSMP needs to know the sparseness of the target signal in advance, which is very suitable for us to extract the fault feature frequency.

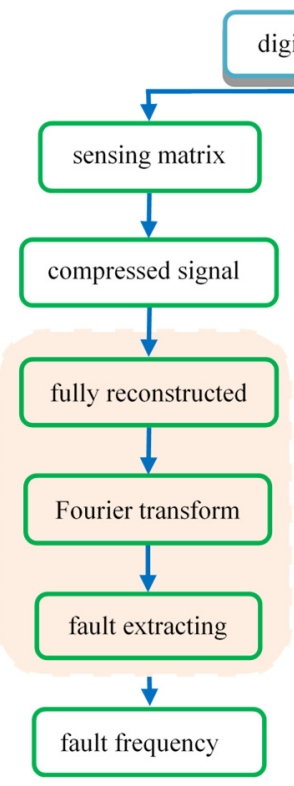

a)

\section{igital signal}

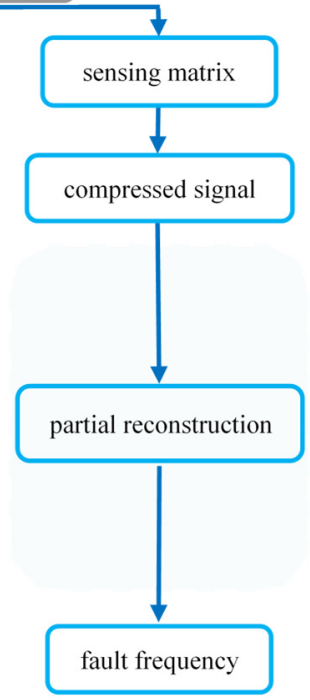

b)

Fig. 4. The compare of fault identification process between whole signal and compressed signal

Table 1. The steps for extracting fault feature frequency from compressed data

\begin{tabular}{|c|}
\hline $\operatorname{CSMP}(\varphi, y, s)$ \\
\hline Input: Sampling matrix $\phi$, compresseddata $y$, sparsity level $s ;$ \\
\hline Output: An $s$-saprse approximation $\alpha$ of the target signal \\
\hline$\alpha^{0} \leftarrow 0$ (Trivial initial approximation) \\
\hline$v \leftarrow y$ (Current samples = input samples) \\
\hline$k \leftarrow 0$ \\
\hline Repeat: \\
\hline$k \leftarrow k+1$ \\
\hline$y \leftarrow \phi^{*} \cdot v$ (From signal proxy) \\
\hline$\Omega \leftarrow s u p p\left(y_{2 k}\right)$ (Identify large components) \\
\hline$T \leftarrow \Omega \cup \operatorname{supp}\left(a^{k-1}\right)$ (Merge supports) \\
\hline$\left.b\right|_{T} \leftarrow \phi_{T}^{-1} \cdot y$ (Signal estimation by least-squares) \\
\hline$\left.b\right|_{T^{c}} \leftarrow 0$ \\
\hline$a^{k} \leftarrow b_{S}$ (Prune to obtain next approximation) \\
\hline$v \leftarrow y-\phi \cdot a^{k}$ (Update current samples) \\
\hline Until halting criterion true \\
\hline
\end{tabular}

The steps for extracting fault feature frequency from compressed data are shown in Table 1 (Algorithm 1. CSMP recovery algorithm). 
For the problem of weak feature identification under noise in mechanical fault diagnosis, the paper proposes a new method of weak feature identification based on CS and SR. In the method, we use stochastic resonance system to enhance the fault frequency and weaken the noise, so the sparseness of the signal gets better, and it is suitable to apply it to compressed sensing. First, we design a stochastic resonance system to process the vibration signal. Then, sparse transformation and compression sampling were taken. Finally, we use the CSMP algorithm to reconstruct the fault feature frequency from the compressed data. The flow chart of the method is shown in Fig. 5.

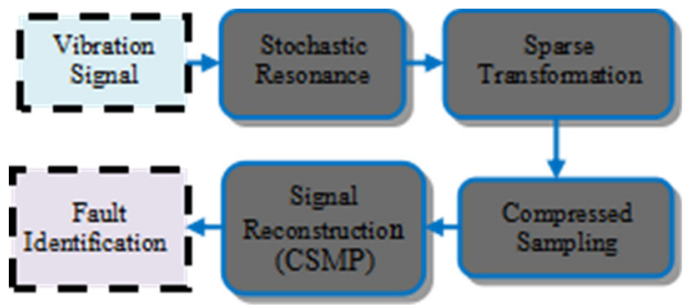

Fig. 5. The workflow of the new method of the paper

\section{Experiment and results}

In this section, two experiments are performed with fault rings of roller element bearings to verify the effectiveness of the proposed method.

\subsection{Inner ring Fault identification of a roller bearing}

The original data of this case is come from the rolling bearing fault simulation platform of Case Western Reserve University [23]. As shown in Fig. 6, the test platform consists of a $2 \mathrm{hp}$ motor (left), a torque transducer/encoder (center), a load motor (right). The test platform bearing comprises a drive end bearing and a fan end bearing. The bearing type of the motor housing used in the experiment is SKF6205, the bearing speed is $N=1797 \mathrm{r} / \mathrm{min}$, the sampling frequency is $12 \mathrm{kHz}$. The status is the inner ring fault and the other information is shown in Table 2.

Table 2. The dimensions of the drive roller bearings

\begin{tabular}{|c|c|c|c|c|c|}
\hline $\begin{array}{c}\text { Inner diameter } \\
/ \mathrm{mm}\end{array}$ & $\begin{array}{c}\text { Outer diameter } \\
/ \mathrm{mm}\end{array}$ & $\begin{array}{c}\text { Pitch diameter } \\
D / \mathrm{mm}\end{array}$ & $\begin{array}{c}\text { Contact angle } \\
\beta /\left(^{\circ}\right)\end{array}$ & $\begin{array}{c}\text { Number of } \\
\text { balls } n\end{array}$ & $\begin{array}{c}\text { Balls diameter } \\
d / \mathrm{mm}\end{array}$ \\
\hline 25.001 & 51.999 & 39.040 & 0 & 9.000 & 7.940 \\
\hline
\end{tabular}

According to the formula:

$f_{i}=\frac{n N}{120 d}\left(1+\frac{d \cos \beta}{D}\right)$.

The theoretical value of the fault feature frequency of the inner ring $f_{i}=162.18 \mathrm{~Hz}$. Fig. 7 presents the waveform and spectra of bearing inner ring fault signal. Fig. 7(a) shows the waveform which exists a fault feature and the sparseness of the signal is poor. In Fig. 7(b), the spectral energy distributes over a wide frequency range and the fault feature frequency is submerged by other frequency signals. What's more, the sparseness is poor too. So, it's necessary to use SR to process the signal. After analyzing the fault feature frequency, we set the coefficients $a=1, b=5$, $D=0.2$, and we use this system to process the vibration signal.

We use the proposed method to identify the fault signal. Fig. 8(a) shows the waveform after stochastic resonance. Fig. 8(b) shows the spectrum, which is also the sparse signal after sparse transformation. It's clear that the fault feature frequency is enhanced, and the sparseness of the signal has been improved. It becomes feasible to use the CS to sample and then extract the fault feature frequency. 


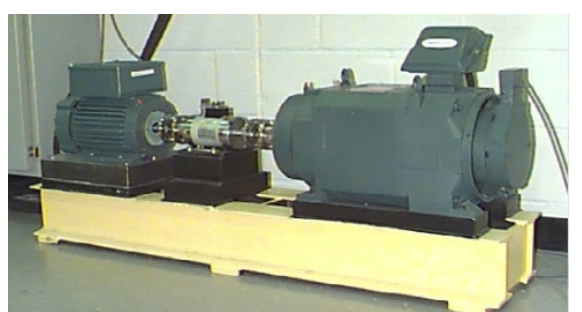

Fig. 6. Rolling bearing test platform of West University

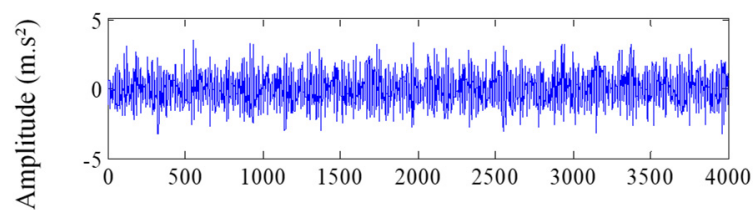

a) Data sequence

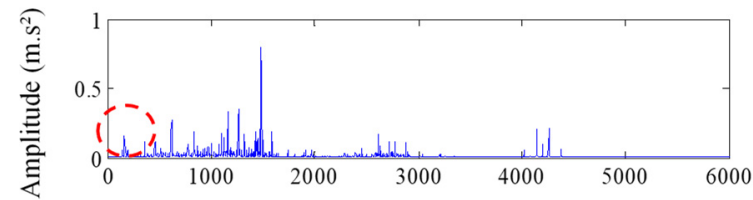

b) Frequency $(\mathrm{Hz})$

Fig. 7. The waveform and spectrum of bearing inner ring fault signal:

a) the waveform, b) the spectrum

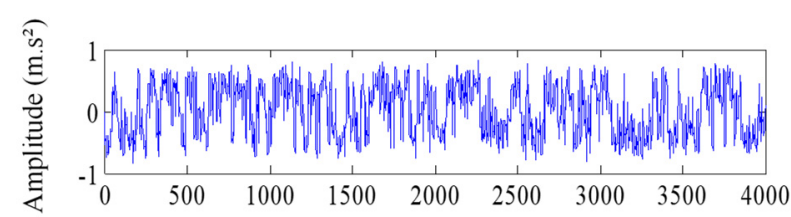

a) Data sequence

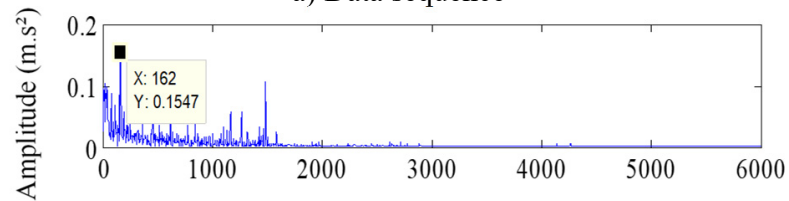

b) Frequency $(\mathrm{Hz})$

Fig. 8. The waveform and spectrum after SR processing: a) the waveform after SR, b) the spectrum after SR

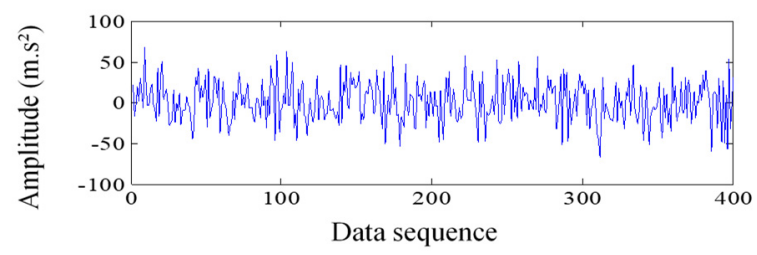

Fig. 9. Compressed sampling signal

In this step, we select a compressible sampled signal section from that of Fig. 8 (a), which is presented in Fig. 9, with 400 samples compared to 4000 in Fig. 8. Here the sampling is not in a tradition way. Instead, it is obtained by multiplying the observation matrix, which is shown in Eq. (2). In this case, $\psi$ is a Fourier matrix of $4000 \times 4000$ and $\phi$ is a random matrix of $400 \times 4000$. 
Next, it is time to extract the fault feature frequency.

In Fig. 10, the extracted signal is shown. Fig. 10(a) shows the sparse signal, which is recovered from the compressed data using CSMP algorithm. It is clear that the signal only contains the fault feature frequency component of $162 \mathrm{~Hz}$. Fig. 10(b) shows waveform of extracted signal. This experiment presents that the proposed method is feasible.

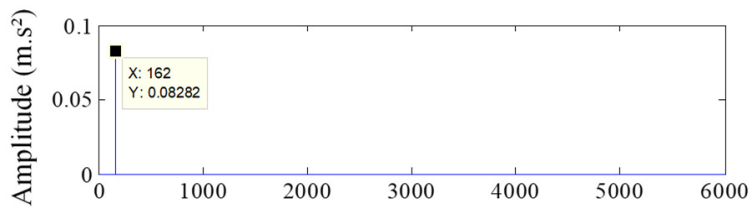

a) Frequency $(\mathrm{Hz})$

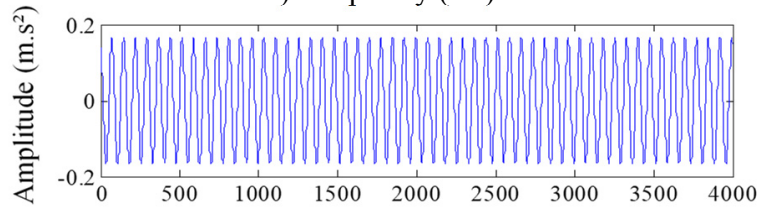

b) Data sequence

Fig. 10. The extracted fault feature signal: a) the frequency spectrum of extracted signal, $b$ ) the waveform of extracted signal

\subsection{Outer ring fault identification of roller bearing}

The experimental data is come from the middle speed shaft outer ring of rotating machinery fault diagnosis test platform, which is shown in Fig. 11. In this case, the bearing model is ER-16K, and we select the data of working condition 29. The measuring point is the middle speed end near the motor end. The speed ratio and load ratio are $80 \%$. The fault feature frequency characteristic of the middle speed shaft of the working condition 29 is $41.435 \mathrm{~Hz}$. In the experiment, the maximum speed of the motor is $3000 \mathrm{rpm}$, the maximum frequency is $50 \mathrm{~Hz}$, the motor speed is calculated: $3000 \times 80 \%=2400$, and the sampling frequency is $12 \mathrm{kHz}$. In Fig. 12, the bearing outer ring fault signal is presented. Fig. 12(a) shows the waveform and Fig. 12(b) shows the spectrum of the vibration signal. It is obvious that fault feature information is submerged in the noise and there is no way to identify the fault feature. What's more, the sparseness of the signal is poor not only in time domain but also in frequency domain. So, it is necessary to use stochastic resonance to process the vibration signal. In this case, we set the coefficients $a=1, b=5$, $D=0.2$.

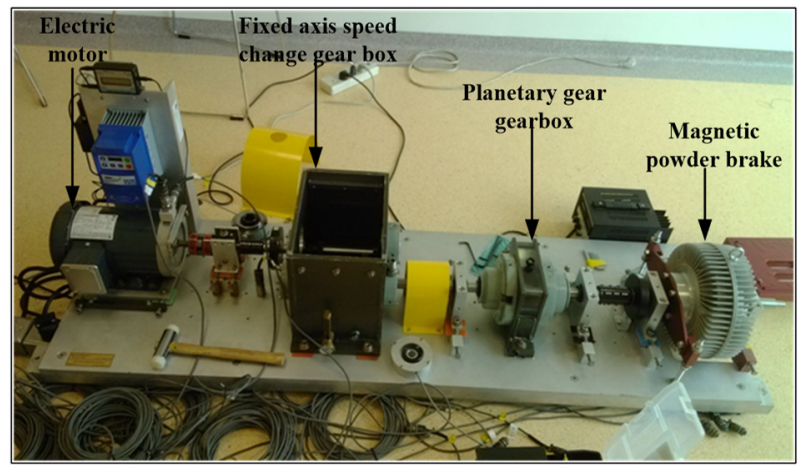

Fig. 11. The test platform

Fig. 13(a) shows the waveform after stochastic resonance while Fig. 13(b) presents the spectrum. It's clear that the fault feature frequency is enhanced, and the sparseness of the signal 
has been improved. It becomes feasible to use the CS to sample and then extract the fault feature frequency.

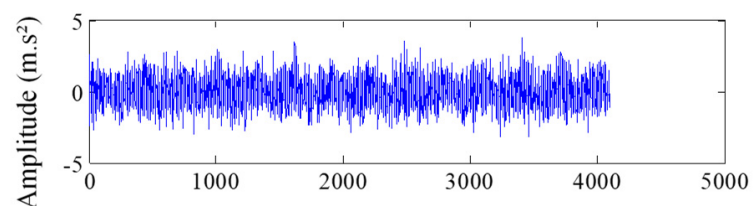

a) Data sequence

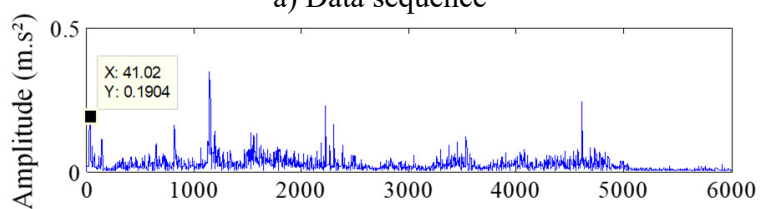

b) Frequency $(\mathrm{Hz})$

Fig. 12. The waveform and spectra of bearing outer ring fault signal: a) the waveform of original signal, b) the spectrum of original signal

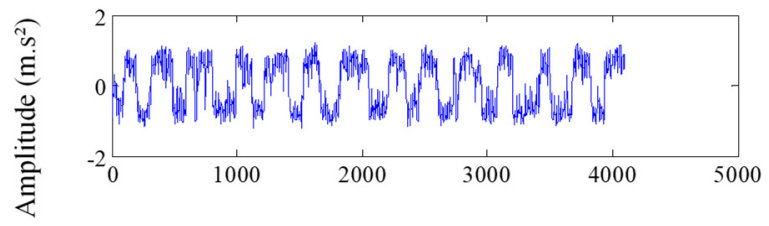

a) Data sequence

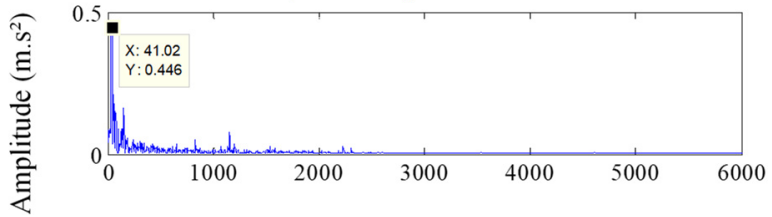

b) Frequency $(\mathrm{Hz})$

Fig. 13. The waveform and spectra of the signal after stochastic resonance processing:

a) the output waveform of SR, b) the spectrum of SR

Same as case 1, the compressed data $y$ is shown in Fig. 14, with 400 sample points compared to 4000 in Fig. 13. It is obtained by multiplying the observation matrix, which is shown in Eq. (2). In this case, $\psi$ is a Fourier matrix of $4000 \times 4000$ and $\phi$ is a random matrix of $400 \times 4000$.

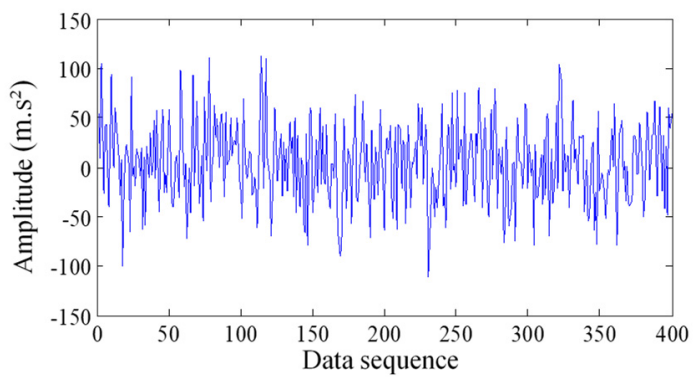

Fig. 14. Compressed sampling for fault diagnosis

Fig. 15 shows the extracted signal. Fig. 15(a) shows the sparse signal, which is recovered from the compressed data using CSMP algorithm. Fig. 15(b) presents the waveform of extracted signal. It is clear that the signal only contains frequency of $42 \mathrm{~Hz}$, which is very close to the fault feature frequency of $41.435 \mathrm{~Hz}$. This case presents that the proposed method is feasible. 


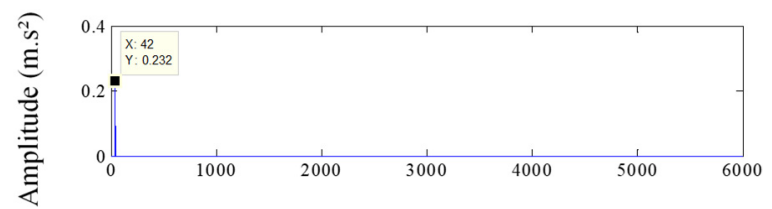

a) Frequency $(\mathrm{Hz})$

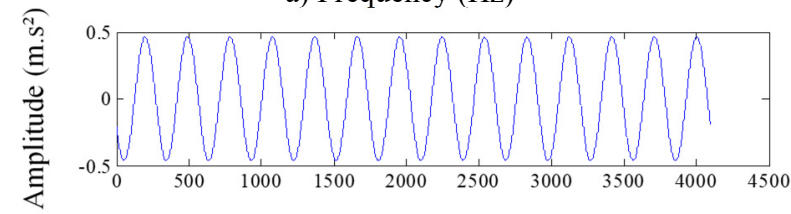

b) Data sequence

Fig. 15. The extracted signal of fault feature frequency: a) the frequency spectrum of extracted signal, b) the waveform of extracted signal

\section{Conclusions}

In this paper, a weak fault identification method is proposed based on CS theory and SR. The machine fault vibration signals are pretreated by stochastic resonance. By this way, the fault signal drowned by noise is amplified and the sparseness of the signals is enhanced, which make it possible to apply compressed sensing. One most outstanding advantage of the method is to extract the mechanical fault feature directly from the compressed data without recovering completely, which reduces the dimensionality of the measurement data and the complexity of algorithm. Another advantage of the method is to defect fault feature directly from far fewer measurements than the Shannon sampling theory requires. The effectiveness of the method has been validated by two experiments.

\section{Acknowledgements}

This work was supported by the National Natural Science Foundation of China (Grant No. 51475407, 51605419), China Postdoctoral Science Foundation (Grant No. 2016M600193) and Training Fund of Talent Project in Hebei Province (Grant No. A2016002018).

\section{References}

[1] Liu J., Shao Y. Overview of dynamic modelling and analysis of rolling element bearings with localized and distributed faults. Nonlinear Dynamics, Vol. 93, 2018, p. 1765-1798.

[2] Naiquan S. U., Jianbin X., Qinghua Z., et al. Research methods of the rotating machinery fault diagnosis. Machine Tool and Hydraulics, Vol. 46, Issue 7, 2018, p. 133-139.

[3] Jianqing Y. U., Guanjian Z., Shikun X., et al. The summary of signal processing technology in fault diagnosis for rotating machinery. Machine Tool and Hydraulics, Vol. 39, Issue 24, 2011, p. 107-110.

[4] Ferguson D., Catterson V. Big data techniques for wind turbine condition monitoring. European Wind Energy Association Annual Event, 2014.

[5] Donoho D. L. Compressed sensing. IEEE Transactions on Information Theory, Vol. 52, Issue 4, 2006, p. 1289-1306.

[6] Candes E. J., Tao T. Near-optimal signal recovery from random projections: universal encoding strategies. IEEE Transactions on Information Theory, Vol. 52, Issue 12, 2006, p. 5406-5425.

[7] Candè E. J., Wakin M. B. An introduction to compressive sampling. IEEE Signal Processing Magazine, Vol. 25, Issue 2, 2008, p. 21-30.

[8] Wang H., Ke Y., Luo G., et al. Compressed sensing of roller bearing fault based on multiple down-sampling strategy. Measurement Science and Technology, Vol. 27, Issue 2, 2016, p. 025009.

[9] Du Z., Chen X., Zhang H. Feature identification with compressive measurements for machine fault diagnosis. Instrumentation and Measurement Technology Conference, 2015.

[10] Tang G., Hou W., Wang H., et al. Compressive sensing of roller bearing faults via harmonic detection from under-sampled vibration signals. Sensors, Vol. 15, Issue 10, 2015, p. 25648. 
[11] Yuan H., Lu C. Rolling bearing fault diagnosis under fluctuant conditions based on compressed sensing. Structural Control and Health Monitoring, Vol. 24, Issue 5, 2017, p. e1918.

[12] Tang G., Yang Q., Wang H. Q., et al. Sparse classification of rotating machinery faults based on compressive sensing strategy. Mechatronics, Vol. 31, 2015, p. 60-67.

[13] Benzi R., Sutera A., Vulpiani A. The mechanism of stochastic resonance. Journal of Physics A: Mathematical and General, Vol. 14, Issue 11, 1999, p. L453.

[14] Mcdonnell M. D., Abbott D. What is stochastic resonance? Definitions, misconceptions, debates, and its relevance to biology. PLOS Computational Biology, Vol. 5, Issue 5, 2009, p. e1000348.

[15] Benzi R. Stochastic resonance: from climate to biology. Nonlinear Processes in Geophysics, Vol. 17, Issue 5, 2007, p. 431-441.

[16] Gammaitoni L., Hanggi P., Jung P., et al. Stochastic resonance: a remarkable idea that changed our perception of noise. European Physical Journal B, Vol. 69, Issue 1, 2009, p. 1-3.

[17] Han D., Li P., An S., et al. Multi-frequency weak signal detection based on wavelet transform and parameter compensation band-pass multi-stable stochastic resonance. Mechanical Systems and Signal Processing, Vol. 70, Issue 71, 2016, p. 995-1010.

[18] Li J., Chen X., He Z. Multi-stable stochastic resonance and its application research on mechanical fault diagnosis. Journal of Sound and Vibration, Vol. 332, Issue 22, 2013, p. 5999-6015.

[19] Qin Y., Tao Y., He Y., et al. Adaptive bistable stochastic resonance and its application in mechanical fault feature extraction. Journal of Sound and Vibration, Vol. 333, Issue 26, 2014, p. 7386-7400.

[20] Zhang X., Hu N., Hu L., et al. Enhanced detection of bearing faults based in signal cepstrum pre-whitening and stochastic resonance. Journal of Mechanical Engineering, Vol. 48, Issue 23, 2012, p. 83-89.

[21] Candes E. J., Romberg J. Errata for quantitative robust uncertainty principles and optimally sparse decompositions. Foundations of Computational Mathematics, Vol. 7, Issue 4, 2007, p. 529-531.

[22] Needell D., Tropp J. A. CoSaMP: Iterative signal recovery from incomplete and inaccurate samples. Applied \& Computational Harmonic Analysis, Vol. 26, Issue 3, 2009, p. 301-321.

[23] Case Western Reserve University Bearing Data Center Website. $\mathrm{http} / / /$ csegroups.case.edu/bearingdatacenter/home.

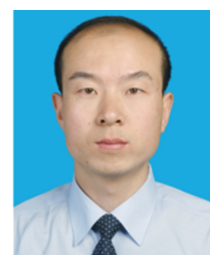

Peiming Shi received Ph.D. degree in Information Science and Engineering Institute from Yanshan University, Qinhuangdao, China, in 2009. Now he is a Professor in Institute of Electrical Engineering of Yanshan University. His current research interests include fault diagnosis and signal processing.

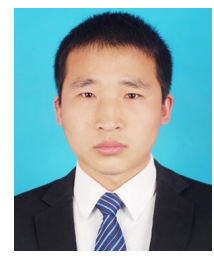

Xiaojie Ma received Bachelor's degree in Electrical Engineering Institute from Yanshan University, Qinhuangdao, China, in 2016. Now he is a postgraduate student in Yanshan University. His current research interests include machinery fault diagnosis.

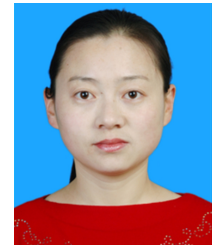

Dongying Han received Ph.D. degree in Mechanical Engineering Institute from Yanshan University, Qinhuangdao, China, in 2008. Now she is an Associate Professor in Institute of Vehicles and Energy of Yanshan University. Her current research interests include fault diagnosis and signal processing. 